\title{
Pre-hospital anaphylaxis severity and the applied therapy
}

\author{
Hajriz Alihodžić ${ }^{1,2,}$, , Devleta Hadžićs,2, Hariz Alihodžić2 \\ ${ }^{1}$ Emergency medical department, Public Institution Health Centre 'Dr. Mustafa Šehović', Tuzla, Bosnia and \\ Herzegovina \\ 2 University of Tuzla, Faculty of Medicine, Tuzla, Bosnia and Herzegovina \\ ${ }^{3}$ Pediatric Clinic, Tuzla University Clinical Centre, Tuzla, Bosnia and Herzegovina
}

Aim: Rapid diagnosis of anaphylaxis and prompt, appropriate therapy are a prerequisite for saving life. The aim of this study was to examine the prehospital method of diagnosis, assessment of the severity of anaphylaxis and choice of therapeutic procedures. Materials and methods: The study involved 182 patients in the Tuzla area with acute allergic reactions that met the clinical criteria for the 2006 diagnosis of anaphylaxis NIAID / FAAN (National Institute of Allergy and Infectious Diseases / Food Allergy and Anaphylaxis Network). Based on the site of treatment, in emergency medical service and primary health care clinics, patients were divided into two groups. The frequency of symptoms, the applied drugs and the final outcome of the prehospital treatment were compared. Results: A total of 182 patients were analyzed, primary health care clinics $N=118(64.8 \%)$, emergency medical service $N=64$ $(35.2 \%)$. Cardiovascular symptomatology of anaphylaxis was more represented in emergency medical emergency medical department $(p=0.024)$. Adrenaline was administered at 9 $(14.1 \%)$ patients with emergency medical care, in primary health care clinics at $22(18.6 \%)$. Statistically is a significant difference in relation to the place of treatment. Statistically higher number of patients in emergency medical care had fluid replacement, antihistamines and glucocorticoids applied $(p<0.05)$. Larger number of patients in emergency medical care had a volume recovery, antihistamines, glucocorticoids $(p<0.05)$. Larger number of patients in emergency medical care had a volume recovery, antihistamines, glucocorticoids ( $p<0.05)$. In emergency medical department had prehospital finished treatment of anaphylaxis 53 patients with anaphylaxis (82.8\%), in primary health care clinics had 30 (25.4\%). Statistically a significant number of patients from primary health care clinics refer to clinical-hospital treatment $(p<0.05)$. Conclusion: Due to different presentation and a small number of standardized diagnostic criteria, there is no unique approach in diagnosing and treating anaphylaxis. Adrenaline must be a medication of choice in anaphylaxis. Future researches should provide precise but simple guidelines for diagnosis and therapy of anaphylaxis.
Table 1. Symptoms listed by organ criteria for the diagnosis of anaphylaxis $(n=182)$

\begin{tabular}{|c|c|c|c|}
\hline Symptoms & $\begin{array}{c}\text { Emergency medical } \\
\text { services } \\
\mathrm{n}=64(\%)\end{array}$ & $\begin{array}{c}\text { Family doctors } \\
\text { practice } \\
\mathrm{n}=118(\%)\end{array}$ & $\mathrm{p}$ value \\
\hline Skin & $57(89,1)$ & $106(89,8)$ & $\mathbf{1 , 0}$ \\
\hline Generalized itching & $46(71,8)$ & $92(77,9)$ & \\
\hline Generalized flushing/erythema & $16(25,0)$ & $58(49,1)$ & \\
\hline Generalized urticaria & $41(64,1)$ & $34(28,8)$ & \\
\hline $\begin{array}{l}\text { Subcutaneous } \\
\text { tissue and mucosa }\end{array}$ & $48(75,0)$ & $93(78,8)$ & 0,69 \\
\hline Localized angioedema & $21(32,8)$ & $54(45,8)$ & \\
\hline Generalized angioedema & $27(42,2)$ & $39(33,0)$ & \\
\hline Respiratory tract & $31(48,4)$ & $52(44,1)$ & 0,16 \\
\hline Sensation of throat tightness & $27(42,2)$ & $38(32,2)$ & \\
\hline Throat itching & $13(20,0)$ & $14(11,9)$ & \\
\hline Hoarseness & $27(42,2)$ & $24(20,3)$ & \\
\hline Wheezing/bronchospasm & $31(48,4)$ & $46(38,9)$ & \\
\hline Gastrointestinal tract & $16(25,0)$ & $42(35,6)$ & 0,19 \\
\hline Dysphagia & $9(14,1)$ & $26(22,0)$ & \\
\hline Abdominal pain & $16(25,0)$ & $30(25,4)$ & \\
\hline Nausea & $6(9,4)$ & $12(10,2)$ & \\
\hline Vomiting & $11(17,2)$ & $22(18,6)$ & \\
\hline Diarrhea & $9(14,1)$ & $13(11,0)$ & \\
\hline Cardiovascular system & $21(32,8)$ & $20(16,9)$ & 0,024 \\
\hline Tachycardia $^{\mathrm{a}}$ & $18(28,1)$ & $12(10,2)$ & \\
\hline Mild Hypotension ${ }^{\mathrm{b}}$ & $12(18,7)$ & $15(12,7)$ & \\
\hline Severe hypotension ${ }^{\mathrm{c}}$ & $7(10,9)$ & $7(5,9)$ & \\
\hline Urinary or fecal incontinence & $4(6,2)$ & $3(2,5)$ & \\
\hline Central Nervous System & $17(26,5)$ & $23(19,5)$ & 0,36 \\
\hline Uneasiness & $2(3,1)$ & $5(4,2)$ & \\
\hline Altered mental status & $7(10,9)$ & $12(10,2)$ & \\
\hline Dizziness & $7(10,9)$ & $12(10,2)$ & \\
\hline Loss of consciousness & $3(4,7)$ & $5(4,2)$ & \\
\hline
\end{tabular}

Table 2. Prehospital therapies of anaphylaxis Emergency medical services and Family doctors practice $(n=182)$

\begin{tabular}{|lccc|}
\hline Treatment & $\begin{array}{c}\text { Emergency medical } \\
\text { services } \\
\mathrm{n}=64(\%)\end{array}$ & $\begin{array}{c}\text { Family doctors } \\
\text { practice } \\
\mathrm{n}=118(\%)\end{array}$ & p value \\
\hline Adrenaline & & & \\
Intravenous fluids & $9(14,1)$ & $22(18,6)$ & 0,30 \\
Antihistamines H1 & $45(70,3)$ & $44(37,3)$ & $<\mathbf{0 , 0 0 1}$ \\
Glucocorticoids & $62(96,9)$ & $71(60,2)$ & $\mathbf{0 , 0 0 4}$ \\
Intravenous Ranitidine & $63(98,4)$ & $98(83,0)$ & 0,02 \\
Inhalative Salbutamol & $62(96,9)$ & $2(1,7)$ & $<\mathbf{0 , 0 0 1}$ \\
Intravenous Aminophyllin & $9(14,1)$ & $12(10,2)$ & 0,83 \\
& $18(28,1)$ & $7(5,9)$ & $<\mathbf{0 , 0 0 1}$ \\
\hline
\end{tabular}

Table 3. Outcome of pre- hospital treatment of anaphylaxis in Emergency medical services and Family doctors practice $(n=182)$

\begin{tabular}{|lccc|}
\hline $\begin{array}{c}\text { Outcome } \\
\text { treatment }\end{array}$ & $\begin{array}{c}\text { Emergency medical } \\
\text { services } \\
\mathrm{n}=64(\%)\end{array}$ & $\begin{array}{c}\text { Family doctors } \\
\text { practice } \\
\mathrm{n}=118(\%)\end{array}$ & p value \\
\hline $\begin{array}{l}\text { Pre-hospital } \\
\text { completed }\end{array}$ & $53(82,8)$ & $30(25,4)$ & $<\mathbf{0 , 0 0 1}$ \\
Clinical & $5(7,8)$ & $27(22,9)$ & $<\mathbf{0 , 0 0 1}$ \\
Hospitalization & $6(9,4)$ & $61(51,7)$ & $<\mathbf{0 , 0 0 1}$ \\
\hline
\end{tabular}

